\title{
Temperature Variation of Debye-Waller Factor for Alkali and Noble Metals
}

\author{
A. R. Jani and V. B. Gohel* \\ Department of Physics, Sardar Patel University, Vallabh Vidyanagar 388 120, Gujarat, India
}

Z. Naturforsch. 38 a, 503-508 (1983); received February 1, 1983

\begin{abstract}
Debye-Waller factors at different temperatures of four alkali and three noble metals have been computed on the basis of a screened shell phenomenological model. The theoretical values are compared with existing experimental data. Particularly for lithium and potassium, most recent experimental information has been included. A critical examination of the results reveals a satisfactory agreement between the theoretical and experimental findings.
\end{abstract}

\section{Introduction}

Studies of the temperature dependence of the intensity of X-ray and neutron-scattering and the Mössbauer effect can be envisaged for obtaining the Debye-Waller factor $\exp (-2 W)$, some times also called the temperature factor because of its strong temperature dependence. Due to the direct relation of this factor with the mean square displacement of the atoms it is obviously relevant for several phenomena of solid state physics such as electrical conductivity and melting. The estimation of this factor on the basis of the ancient Debye model being not satisfactory [1], more realistic lattice dynamical models are needed.

During recent years the Debye-Waller factor obtained from experiments has often been compared with that resulting from calculations [2-29]. These calculations have been carried out for cubic metals with the help of phenomenological models [30-34] incorporating various radial and angular force constants. It is evident that such comparison provide a test of the models used.

Recently, we have put forth a screened shell phenomenological model for lattice dynamics of cubic metals [35]. This model was then modified for b.c.c. transition metals to compute the phonon dispersion relations in the symmetry directions [36]. As our models have explained the dispersion relations of alkali, noble and transition metals quite satisfac-

* Department of Physics, School of Science, Gujarat University, Ahmedabad 380009 Gujarat, India.

Reprint requests to Dr. A. R. Jani, Department of Physics, Sardar Patel University, Vallabh Vidyanagar 388120, Gujarat, India. torily, we thought it worthwhile to examine its applicability for other properties also. In the present paper we have studied the temperature variation of the Debye-Waller factor, the Debye characteristic temperature and the mean square atomic displacement for sodium, rubidium, potassium, lithium, copper, silver and gold on the basis of our model [35].

\section{Theory}

In the harmonic approximation, the Debye-Waller exponent $2 W$ is directly related with the mean square displacement of the atoms, and following James [37] it can be given as

$$
\begin{aligned}
2 W= & \frac{h}{4 \pi^{2} M N} \sum_{\boldsymbol{q}, j} \frac{\left(\boldsymbol{K} \cdot \hat{e}_{\boldsymbol{q}, j}\right)^{2}}{v_{\boldsymbol{q}, j}} \\
& \cdot\left\{\frac{1}{2}+\frac{1}{\exp \left\{\frac{h v_{\boldsymbol{q}, j}}{k_{\mathrm{B}} T}\right\}-1}\right\},
\end{aligned}
$$

where $M$ is the mass of the atom, $N$ the total number of unit cells in the crystal, $K$ the difference of the initial and final wave vectors of X-rays, $v_{\boldsymbol{q}, j}$ the frequency of a phonon with the wave vector $\boldsymbol{q}$ and polarization $j, \hat{e}_{q . j}$ the polarization vector of the $(\boldsymbol{q}, j)$ lattice mode, and the summation extends over the all normal modes.

For a monatomic cubic crystal, the polarization factor $\left(\boldsymbol{K} \cdot \hat{e}_{\boldsymbol{q} . j}\right)^{2}$ can be replaced by its average value outside the summation so that (1) becomes

$$
2 W=\frac{2 h}{3 M N}\left(\frac{\sin \theta}{\lambda}\right)^{2} \sum_{\boldsymbol{q}, j} \frac{1}{v_{\boldsymbol{q}, j}} \operatorname{coth}\left\{\frac{h v_{\boldsymbol{q}, j}}{2 k_{\mathrm{B}} T}\right\} .
$$


If $g(v)$ is the frequency distribution function for the phonon giving the number of vibrational modes in the frequency interval between $v$ and $v+\mathrm{d} v$, (2) becomes

$2 W=\frac{8 \pi^{2} \hbar}{3 M N}\left(\frac{\sin \theta}{i}\right)^{2} \int_{0}^{r_{\max }} \frac{g(v)}{v} \operatorname{coth}\left\{\frac{h v}{2 k_{\mathrm{B}} T}\right\} \mathrm{d} v$,

where $\theta$ is the glancing angle, and $\lambda$ the wavelength of the incident radiation.

The Debye-Waller factor is now calculated from (3). For a Debye model of the solid, the temperature dependence of the Debye-Waller exponent can be written as

$$
2 W=\frac{48 \pi^{2} \hbar^{2} T}{M k_{\mathrm{B}} \theta_{\mathrm{M}}^{2}}\left\{\Phi(x)+\frac{x}{4}\right\}\left(\frac{\sin \theta}{i}\right)^{2},
$$

where $\theta_{\mathrm{M}}$ is the effective X-ray characteristic temperature, $\Phi(x)$ is the usual Debye integral function, and $x=0)_{\mathrm{M}} / T$.

Similarly, in the harmonic approximation, the mean square displacement of the atoms is given by the relation

$$
\bar{u}^{2}=\frac{3}{8 \pi^{2}}\left(\frac{i}{\sin \theta}\right)^{2} W .
$$

\section{Numerical Computation}

To compute the Debye-Waller exponent $2 W$, it is essential to determine the frequency spectrum of the lattice vibrations at a suitable number of points in an irreducible section of the first Brillouin zone. This frequency distribution has been obtained [38] by the numerical sampling of frequencies according to Blackmann's technique [39-40] over a discret subdivision in the wave vector space.

In order to have a large survey of frequencies, in the present work we have considered a mesh of evenly spaced 64000 wave vectors in the first Brillouin zone. For this purpose, the first Brillouin zone was divided into $40 \times 40 \times 40$ miniature cells with axes $1 / 40$ of the length of the reciprocal lattice cell. Consideration of Born's cyclic boundary condition and symmetry property of the lattice reduces the 64000 points to 1661 non-equivalent points lying within an irreducible 1/48 th part of the first Brillouin zone. Each point was weighted according to the number of points equivalent to it by symmetry. Care was taken in giving the proper weights to points lying on the surfaces, edges and corners of the Brillouin zone. The 1,92,000 phonon eigenfre- quencies corresponding to 64000 eigenvectors in the zone were computed from the solution of the secular determinant formed by the dynamical matrix due to our proposed theory [35]. To evaluate the frequency distribution function $g(v)$, counts of the number of frequencies falling in to intervals of a 100th part of the maximum frequency were used to construct a frequency histogram. It should be emphasized that in most of the previous calculations [2-28] a much coarser mesh has been used.

The derivation of the dynamical matrix, its diagonalization and hence the frequency wave vector dispersion relations, the elastic constants and other pertinent data for the metals studied in the present work have already been given and discussed in our previous papers $[35,41-43]$.

\section{Results and Discussion}

The comparison between theoretical and available experimental data is made in terms of the temperature parameter $Y$ given by

$$
Y=\log _{10} e\left(\frac{i}{\sin \theta}\right)^{2}\left(2 W_{T_{0}}-2 W_{T}\right),
$$

where $2 W_{T}$ and $2 W_{T_{0}}$ are the values of the DebyeWaller exponents for the temperatures $T$ and $T_{0}$, respectively. This quantity is independent of $\lambda$ and (), and is directly accessible from the measured intensity of the Bragg reflection in X-ray diffraction experiments. If $I_{T}$ and $I_{T_{0}}$ are the measured integrated intensities of a certain diffraction line at the temperatures $T$ and $T_{0}$, respectively, we have

$$
I_{T} / I_{T_{0}}=\exp \left(-2 W_{T}\right) / \exp \left(-2 W_{T_{0}}\right) .
$$

This gives

$$
\begin{aligned}
Y & =\left(\frac{i}{\sin \theta}\right)^{2} \log _{10}\left(I_{T} / I_{T_{0}}\right) \\
& =\log _{10} e\left(\frac{i}{\sin \theta}\right)^{2}\left(2 W_{T_{0}}-2 W_{T}\right) .
\end{aligned}
$$

The right hand sides of the above equation are evaluated due to (3). The computed values and the experimental results with reference to a standard temperature for sodium, copper, silver and gold are given and discussed here. In the absence of detailed experimental information for lithium, potassium and rubidium, we have tabulated the theoretical results from the present work for a future comparison. 
Table 1. Debye-Waller factors and mean square displacements of atoms for lithium.

\begin{tabular}{lll}
\hline Temperature $(\mathrm{K})$ & $W_{T}(\AA)^{2}$ & $\bar{u}^{2}(\AA)^{2}$ \\
\hline 50 & 1.348 & 0.051 \\
100 & 1.809 & 0.069 \\
150 & 2.394 & 0.091 \\
200 & 3.028 & 0.115 \\
250 & 3.688 & 0.140 \\
300 & 4.361 & 0.166 \\
350 & 5.042 & 0.191 \\
400 & 5.728 & 0.217 \\
450 & 6.417 & 0.244 \\
\hline
\end{tabular}

Corresponding calculated value of $\theta_{\mathrm{M}}$ comes out to be $344.5 \mathrm{~K}$.

Table 2. Debye-Waller factors and mean square displacements of atoms for potassium.

\begin{tabular}{lcl}
\hline Temperature $(\mathrm{K})$ & $W_{T}(\AA)^{2}$ & $\bar{u}^{2}(\AA)^{2}$ \\
\hline 50 & 1.914 & 0.072 \\
100 & 3.591 & 0.136 \\
150 & 5.319 & 0.202 \\
200 & 7.060 & 0.268 \\
250 & 8.807 & 0.334 \\
300 & 10.556 & 0.401
\end{tabular}

The value of $\theta_{M}$ from the above table was found to be $96.6 \mathrm{~K}$.

Table 3. Computed values of Debye-Waller factor and mean square displacement of atoms for rubidium.

\begin{tabular}{lcl}
\hline Temperature $(\mathrm{K})$ & $W_{T}(\AA)^{2}$ & $\bar{u}^{2}(\AA)^{2}$ \\
\hline 50 & 2.743 & 0.104 \\
100 & 5.376 & 0.204 \\
150 & 8.034 & 0.305 \\
200 & 10.697 & 0.406 \\
250 & 13.363 & 0.507 \\
300 & 16.030 & 0.609 \\
\hline
\end{tabular}

For rubidium, $\theta_{\mathrm{M}}$ from the above values comes out to be $55.7 \mathrm{~K}$.

Lithium: Recently, Bednarz and Field [44] have reported an accurate X-ray diffraction investigation on single crystals of lithium in the temperature range $293-423 \mathrm{~K}$. They have obtained $\theta_{\mathrm{M}}=$ $(326 \pm 1) \mathrm{K}$, while Pankow [45] did find $\theta_{\mathrm{M}}=$ $(352 \pm 12) \mathrm{K}$. The value obtained for $\theta_{\mathrm{M}}$ in the present study is $344.5 \mathrm{~K}$. Corresponding values of $W_{T}$ and $\bar{u}^{2}$ at different temperatures due to the present study are tabulated in Table 1.

Sodium: Experimental measurements for sodium in the temperature range $117-368 \mathrm{~K}$ were made by Dawton [46]. He has measured the intensity ratios $I_{117} / I_{T}$ for the chilled crystal for (110), (200), (220), (310), (400) and (440) reflections at three different temperatures. Of these, only the (220), (310) and (400) reflections are reliable. The average values of $Y, \bar{u}^{2}$ and $\theta_{\mathrm{M}}$ obtained from these reflections are displayed in Figure 1. The full lines represent the calculated values. In the present work, $\theta_{\mathrm{M}}$ was found to be $155 \mathrm{~K}$. A comparison of this value with that reported by Singh and Sharma [5] shows that extending the Cheveau model [34] out to second neighbours has a considerable effect on $\theta_{\mathrm{M}}$. Our $\theta_{\mathrm{M}}$ is consistently larger than the experimental values. The discrepancy increases with temperature, which is an obvious fact since our calculations are based on the harmonic approximation. Calculations based on the harmonic approximation should provide a better agreement at low temperatures. This is what has been found in the present study for sodium. It is interesting to note that, contrary to this fact, the theoretical values of $\theta_{\mathrm{M}}$ due to Singh and Sharma [5] show an opposite trend. The reliability of our results can be judged by comparing our $\theta_{\mathrm{M}}$ with the experimental $\theta_{\mathrm{c}}=156.6 \mathrm{~K}$ (Calorimetric $\theta_{\mathrm{c}}$ ) reported by Martin [47].

The agreement between the observed and our calculated $\bar{u}^{2}$ values for low temperatures up to about $200 \mathrm{~K}$ is very satisfactory. The same is the case with the parameter $Y$. It can be inferred from these results that in sodium anharmonic effects begin to become important at about $200 \mathrm{~K}$.

Potassium: Krishnakumar and Viswamitra [48] have measured $W_{T}, \bar{u}^{2}$ and $\theta_{\mathrm{M}}$ by X-ray diffraction at room temperature and obtain $9.55 \AA^{2}, 0.36 \AA^{2}$ and $96 \mathrm{~K}$, respectively. Our calculated values at different temperatures are given in Table 2. It can be seen that our values at $300 \mathrm{~K}$ are very close to the experimental findings. Moreover, very recently [49] accurate relative and absolute integrated intensity measurements in X-ray diffraction experiments on two single crystals of potassium in the temperature range $207-337 \mathrm{~K}$ have been put forth. In this investigation the Debye temperature $(81 \pm 1) \mathrm{K}$ has been predicted at $293 \mathrm{~K}$. Looking to the difference between the experimental data, our theoretical value of $96.6 \mathrm{~K}$ for $\theta_{\mathrm{M}}$ is quite justifiable.

Rubidium: Experimental information for this metal is not available. Our theoretical values of $W_{T}$, $\bar{u}^{2}$ and $\theta_{\mathrm{M}}$ at different temperatures are given in Table 3 .

Copper: The temperature variation of the DebyeWaller factor of copper from the X-ray intensity measurements has been studied by Flinn et al. [50], 

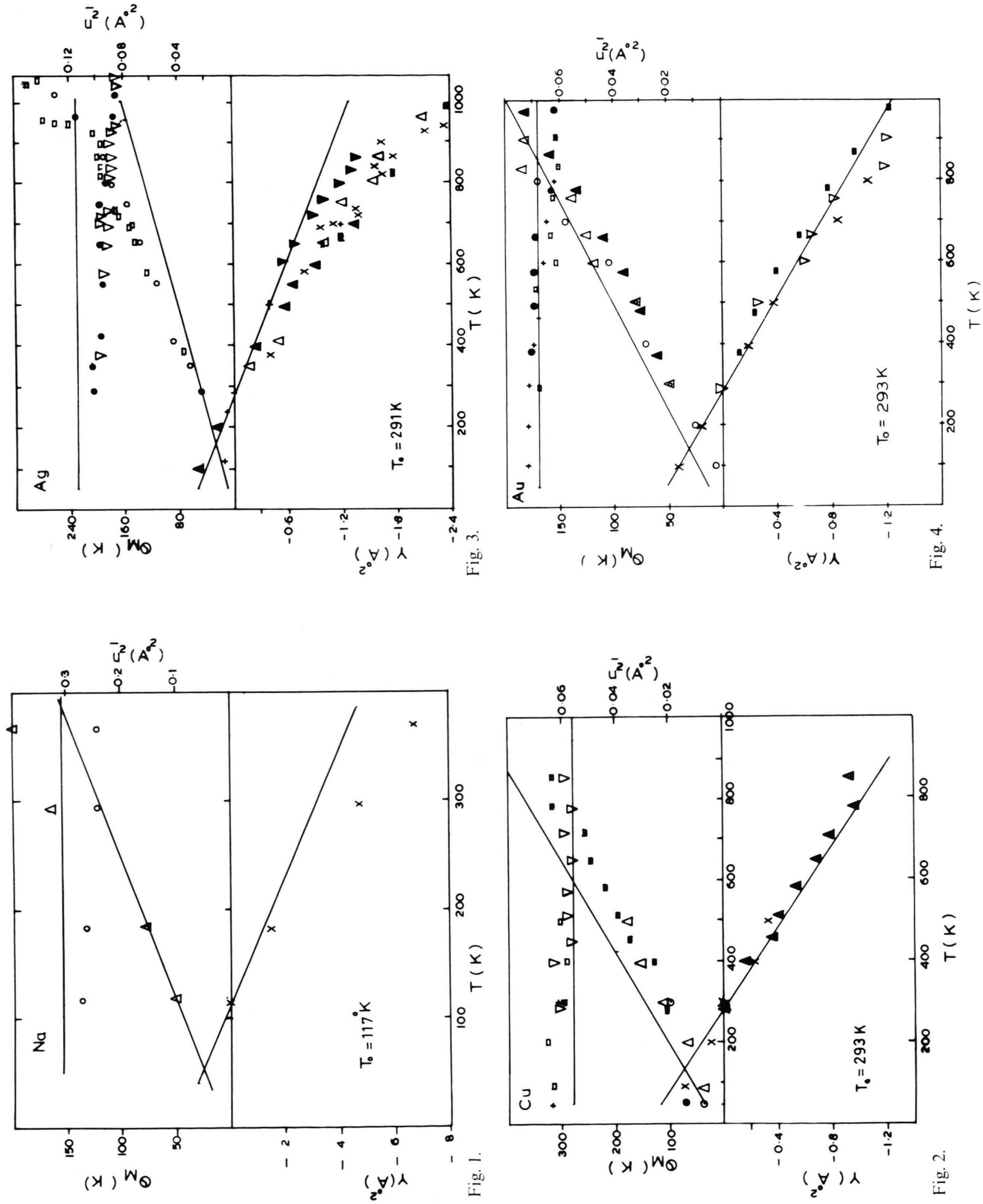
Owen and Williams [51] and Chipman et al. [52]. Flinn et al. [50] have measured the integrated intensities of the (600), (800) and (1000) reflections from a single copper crystal at selected temperatures in the temperature range $4.2-500 \mathrm{~K}$. These authors have expressed their results in terms of the effective Debye temperature $0_{\mathrm{M}}$. Owen and Williams [51] covered the range from room temperature to $900 \mathrm{~K}$ for microphotometric measurements on the lines in $X$-ray structure spectra of powder specimens. They have calculated $Y$ values at selected temperatures. Both measurements are consistent and agree with those of Chipman et al. [52] at room and liquid nitrogen temperatures on copper powder. The values of $Y, \bar{u}^{2}$ and $\theta_{\mathrm{M}}$ deduced from the experimental data of these workers are plotted in Figure 2. Our results are shown by full lines in Figure 2. The agreement between theory and experiment is fairly good.

Silver: Among many measurements by different workers using various techniques [53 - 58] the measurements due to Haworth [56] are considered to be most exhaustive and reliable. His observations cover the temperature range from $286 \mathrm{~K}$ to $1100 \mathrm{~K}$ and are in excellent agreement with those of Simerska [57] who covered the temperature range $291-$ $1033 \mathrm{~K}$. The experimental points reported by the different authors [53-58] are shown in Fig. 3 along with the theoretical full lines. The agreement between the theoretical and experimental values up to about $500 \mathrm{~K}$ is quite satisfactory.

Gold: The experimental results due to different authors [51, 58, 59] for gold are plotted in Fig. 4 along with our computed values. The theoretical results are found to be in broad agreement with the experimental findings. Moreover, particularly for this metal in the family of noble metals we would

Fig. 1. Temperature variation of $Y, \bar{u}^{2}$ and $\theta_{\mathrm{M}}$ for sodium. Experimental values $(\times, \Delta, 0)$ are due to Dawton [46].

Fig. 2. Temperature variation of $Y, \bar{u}^{2}$ and $\theta_{\mathrm{M}}$ for copper. Experimental results are from: $(\bullet, 0,+)$ Chipman and Paskin [52]; $(\times, \Delta, \square)$ Flinn et al. $[50]$ and $(\Delta, \mathbf{\Delta}, \nabla)$ Owen and Williams [51].

Fig. 3. Temperature variation of $Y, \bar{u}^{2}$ and $\theta_{\mathrm{M}}$ for silver. Experimental data are from: $(\triangle, O, \bullet)$ Simerska [57]; $(\times, \square, \nabla)$ Haworth [56]; (+) Boskovits et al. [54]; (ム) Alexopoulos et al. [58]; (ש) Spreadborough and Christian [55] and (๑) Andriessen [53].

Fig. 4. Temperature variation of $Y, \bar{u}^{2}$ and $\theta_{\mathrm{M}}$ for gold. Experimental results are from: $(x, O,+)$ Alexopoulos et al. $[58] ;(\nabla, \circ, \square)$ Owen and Williams [51] and $(\boldsymbol{\bullet}, \boldsymbol{\Delta}, \bullet)$ Synecek et al. [59]. like to point out that our theoretical results are better than those reported in the recent past on the basis of phenomenological theories [14, 17, 23].

We have shown that the Debye-Waller factor and allied parameters evaluated with the help of our theory [35] agree well with the observed temperature variation of the intensities of X-ray reflections at low temperatures. At higher temperatures, the experimental $Y$ versus $T$ curves show a marked nonlinearity; the observed decrease in intensity becomes greater than the theoretical one, and the divergence between the two increases with temperature. The effective X-ray characteristic temperature $\theta_{\mathrm{M}}$, computed in the present work agrees quite well with the experimental values. The theoretical mean square displacements of the atoms fit well with the experimental data up to a certain temperature.

The apparent discrepancy between theory and experiment is not unexpected and can be attributed to the neglect of the temperature variation of the vibrational frequencies [60] and to other anharmonic effects [61-66].

In the present work, no account has been taken of the temperature variation of the elasticity and the lattice parameters. With increase of temperature, the lattice frequencies diminish because of the lattice expansion. This depends upon the Grüneisen parameter which varies with temperature and lattice frequencies. Several attempts [51, 55, 56] have been made to account for this effect in terms of the Grüneisen parameter. However, the available data do not allow for a detailed discussion. It appears that still there is a need of more detailed studies to relate this parameter with the anharmonicity in the lattice vibrations.

At the present level of accuracy, the computed results indicate that our screened shell phenomenological model [35] is quite adequate for a theoretical interpretation of X-ray Debye-Waller factor data of alkali and noble metals.

\section{Acknowledgements}

One of the authors (ARJ) would like to express his appreciation to Professor M. S. Joshi (Head of the Department) and Professor M. K. Agarwal for their support and encouragement to carry out this work. We also thank the Sardar Patel University and the Gujarat University for providing the computer facilities. 
[1] F. H. Herbstein, Adv. Phys. 10,313 (1961).

[2] P. S. Mahesh and B. Dayal, phys. stat. sol. 7, 399 (1964).

[3] P. K. Sharma and K. N. Mehrotra, An. Real. Soc. Espan. Fis. 65, 189 (1969).

[4] A. K. Singh and P. K. Sharma, J. Phys. Soc. Japan 26, 425 (1969).

[5] N. Singh and P. K. Sharma, Phys. Rev. B. 3, 1141 (1971).

[6] P. K. Sharma and S. K. Sangal, Z. Phys. Chem. Leipzig. 247, 257 (1971).

[7] J. Behari and B. B. Tripathi, J. Phys. Soc. Japan. 33, 1207 (1972).

[8] S. Jogi, J. Phys. F. 4, 11 (1974).

[9] K. Sneh, Ind. J. Pure Appl. Phys. 12, 89 (1974).

[10] J. Prakash and M. P. Hemkar, J. Phys. Soc. Japan. 34, 1583 (1973).

[11] S. Chandra, H. L Kharoo, and M. P. Hemkar, Nuovo Cim. 30 B, 1 (1975).

[12] J. Prakash, L. P. Pathak, and M. P. Hemkar, Aust. J. Phys. 28,63 (1975).

[13] O. P. Gupta, J. Phys. Soc. Japan. 38, 1451 (1975).

[14] R. C. Rai and M. P. Hemkar, Ind. J. Pure Appl. Phys. 14, $976(1976)$

[15] L. P. Pathak, V. P. Singh, J. Prakash, and M. P. Hermkar, Ind. J. Phys. 51 A, 42 (1977).

[16] R. P. Gupta and P. K. Sharma, J. Chem. Phys. 46, 1359 (1967).

[17] H. L. Kharoo, O. P. Gupta, and M. P. Hemkar, Z. Naturforsch. 32 a, 570 (1977).

[18] J. P. Dixit and K. N. Mehrotra, Acta. Phys. Acad. Sci. Hung. 42, 127 (1977).

[19] H. L. Kharoo, O. P. Gupta, and M. P. Hemkar, J. Phys. Soc. Japan 43, 2030 (1977)

[20] O. P. Gupta and M. P. Hemkar, Z. Naturforsch. 32 a, 1495 (1977).

[21] O. P. Gupta and M. P. Hemkar, Nuovo Cim. 45 B, 255 (1978).

[22] O. P. Gupta and M. P. Hemkar, Physica $(B+C)$. $94(B+C), 319$ (1978).

[23] M. Kumar and M. P. Hemkar, Acta. Phys. Pol. A. 53 A, 543 (1978).

[24] K. N. Mehrota and J. P. Dixit. Acta Phys. Acad. Sci. Hung 46, 27 (1979).

[25] O. P. Gupta and H. L. Kharoo, Ind. J. Pure Appl. Phys. 17, 245 (1979).

[26] B. P. Singh, L. P. Pathak, and M. P. Hemkar, Z. Naturforsch 35 a, 230 (1980).

[27] R. Cavalheiro and M. M. Shukla, phys. stat. sol. 98 b, 689 (1980).

[28] K. N. Mehrotra, R. G. Tambay, and C. K. Srivastava, Acta Cien. Indica. 6, 42 (1980).

[29] A. R. Jani and V. B. Gohel, Acta Phys. Pol. A, in press 1983.

[30] J. de Launay, Solid Stat. Phys. (AP). 2, 219 (1956).
[31] A. B. Bhatia, Phys. Rev. 97, 363 (1955).

[32] P. K. Sharma and S. K. Joshi, J. Chem. Phys. 39, $2633(1963)$

[33] K. Krebs, Phys. Rev. 138, A 143 (1965).

[34] L. Cheveau, Phys. Rev. 199, 496 (1968).

[35] V. B. Gohel and A. R. Jani, Physica 82 B, 333 (1976).

[36] V. B. Gohel and A. R. Jani, Pramana 6, 593 (1977).

[37] A. R. Jani, Ph. D. Thesis (Gujarat University, India) P 151 (1979).

[38] M. Blackman, Proc. Roy. Soc. London A 195, 416 (1937).

[39] M. Blackman, Encyclopaddeia of Physics, SpringerVerlag, Berlin 7, 325 (1955).

[40] E. I. Geshko, Ubrain. Fiz. Zh. 8, 1058 (1963).

[41] A. R. Jani and V. B. Gohel, Acta Phys. Acad. Sci. Hung. 51, 325 (1981).

[42] A. R. Jani, Spurj, Sardar Patel University Research J. 1, 119 (1982).

[43] A. R. Jani and V. B. Gohel, Solid St. Commun. 43, 945 (1982).

[44] B. Bednarz and D. W. Field, Acta Crystallogr. A 38, 163 (1982).

[45] G. Pankow, Helv. Phys. Acta. 9, 87 (1936).

[46] R. H. V. M. Dawton, Proc. Phys. Soc. 49, 294 (1937).

[47] D. L. Martin, Phys. Rev. 124, 438 (1961).

[48] S. Krishnakumar and M. A. Viswamitra, Phys. Letts. 37 A, 143 (1971).

[49] B. Bednarz and D. W. Field, Acta Crystallographica A 38, 3 (1982).

[50] P. A. Flinn, R. W. McManus, and J. A. Rayne, Phys. Rev. 123,809 (1961).

[51] E. A. Owen and R. W. Williams, Proc. Roy. Soc. 188 A, 509 (1947).

[52] D. R. Chipman and A. Paskin, J. Appl. Phys. 30, 1992 (1959).

[53] R. Andriessen, Physica 2, 417 (1935).

[54] J. Boskovits, M. Roilos, A. Theodossius, and K. Alexopoulos, Acta Crystallogr. 11,845 (1958).

[55] J. Spreadborough and J. W. Christian, Proc. Phys. Soc. 74, 609 (1959)

[56] C. W. Haworth, Phil. Mag. 5, 1229 (1960).

[57] M. Simerska, Acta Crystallogr. 14, 1259 (1961).

[58] K. Alexopoulos, J. Boskovits, S. Mourikis, and M. Roilos, Acta Crystallogr. 19, 349 (1965).

[59] V. Synecek, H. Chessin, and M. Simerska, Acta. Crystallogr. 26 A, 108 (1970).

[60] A. Paskin, Acta. Crystallogr. 10,667 (1957).

[61] H. Hahn and W. Ludwig, Z. Physik 161, 404 (1961)

[62] A. A. Maradudin and P. A. Flinn, Phys. Rev. 129, 2529 (1963).

[63] L. S. Slater, Advan. Phys. 14, 1 (1965).

[64] R. A. Cowley, Advan. Phys. 12, 421 (1963).

[65] G. A. Wolfe and B. Goodman, Phys. Rev. 178, 1171 (1969).

[66] B. T. M. Willis, Acta. Crystallogr. A 25, 277 (1969). 